J. Clin. Chem. Clin. Biochem.

Vol. 20,1982 , pp. $587-591$

$\alpha_{1}$-Antitrypsin: Referenzwerte in Serum und Plasma mit Benzoyl-D, L-arginin-p-nitroanilid, einem chromogenen Substrat

Von I. Witt

Biochemisches Labor der Universitäts-Kinderklinik, Freiburg i.Br. und

\title{
W. Tritschler
}

Boehringer Mannheim GmbH, Diagnostica Forschung, Mannheim

(Eingegangen am 8. Februar/5. Mai 1982)

Zusammenfassung: Mit einer konfektioniert erhältlichen Testpackung für die Bestimmung von $\alpha_{1}$-Antitrypsin in einem kontinuierlichen Verfahren bei $25^{\circ} \mathrm{C}$ wurden Referenzwerte bestimmt. Für ein Kollektiv von 100 Probanden im Alter von 20 bis 60 Jahren ergab sich für Serum ein Referenzbereich von 1,4 bis 2,4 kIU/l (1,9-3,4 g/l), für Plasma von 1,4 bis $2,1 \mathrm{kIU} / 1(1,9-3,0 \mathrm{~g} / 1)$, (jeweils 5 . bis 95 . Perzentil).

Eine Alters- oder Geschlechtsabhängigkeit konnte nicht nachgewiesen werden; präprandiale und postprandiale Aktivitäten unterschieden sich nicht, zwischen Rauchern und Nichtrauchern wurde kein Unterschied der $\alpha_{1}$-AntitrypsinAktivitäten gefunden. Bei den Frauen ließ sich zwischen den Kollektiven mit und ohne hormonale Kontrazeption ein signifikanter Unterschied der Aktivitäten nachweisen.

Berücksichtigt man bei der Berechnung der Aktivitäten im Citratplasma die Verdünnung des Blutes mit Citratlösung, dann zeigt sich, daß in Plasma höhere Werte als in Serum gefunden werden (Median 13\%): Die Messung in Plasma dürfte die in vivo-Aktivität von $\alpha_{1}$-Antitrypsin besser wiedergeben.

$\alpha_{1}$-Antitrypsin: Reference values in serum and plasma determined using benzoyl-D,L-arginine-p-nitroanilide, a chromogenic substrate

Summary: Reference values for $\alpha_{1}$ antitrypsin were determined using a commercially available kit for continuous assay at $25^{\circ} \mathrm{C}$. With a population of 100 probands aged between 20 and 60 years, a reference range of $1.4-2.4 \mathrm{kIU} / 1$ (1.9-3.4 $\mathrm{g} / \mathrm{l})$ was obtained for serum, and 1.4-2.1 kIU $/ 1(1.9-3.0 \mathrm{~g} / \mathrm{l})$ for plasma. In each case, these ranges correspond to the 5 th to 95 th percentile. No variations according to age or sex were observed, nor was any difference noted between preprandial and postprandial activities, or between values in smokers and nonsmokers. Significantly different values were found in the group of women taking oral steroid contraceptives and the group not taking such contraceptives.

If the dilution of the blood sample with citrate solution is taken into account in calculating the activity in citrated plasma, it is apparent that higher values are found in plasma than in serum (median 13\%): Hence, the in vivo$\alpha_{1}$-antitrypsin activity can most probably be better determined using a plasma sample.

\section{Einleitung}

$\alpha_{1}$-Antitrypsin ist ein Akutphasenprotein: Bei entzündlichen Erkrankungen, Tumoren und Gewebszerfall reagiert es mit einer vermehrten Synthese (1). Klinisch wichtiger ist die Assoziation von genetisch bedingtem $\alpha_{1}$-Antitrypsin-Mangel mit dem im Erwachsenenalter auftretenden obstruktiven Lungenemphysem $(1,2)$. Ein weiteres klinisches Korrelat des angeborenen $\alpha_{1}$-Antitrypsin-Mangels ist das Krankheitsbild der juvenilen Lebercirrhose $(3,4,5)$. Die Beurteilung des $\alpha_{1}$-Antitrypsin-Wertes ist dadurch erschwert, daß Abhängigkeiten beschrieben sind: In der Schwangerschaft und bei Einnahme oraler Kontrazeptiva wurden erhöhte Werte gefunden $(1,6,7)$. Weniger gesichert scheinen $\mathrm{Ge}$ schlechts- und Altersabhängigkeiten zu sein $(7,8,9)$. 
Im Zusammenhang mit der Einführung einer Testkombination zur Bestimmung der $\alpha_{1}$-AntitrypsinAktivität haben wir für Serum und Plasma Referenzwerte ermittelt und sind der Frage nach Abhängigkeiten $\operatorname{der} \alpha_{1}$-Antitrypsin-Aktivität nachgegangen.

\section{Material und Methoden}

\section{Testprinzip}

Serum oder Citratplasma werden mit einem Überschuß von Trypsin versetzt. Das nicht gebundene Trypsin setzt aus dem Substrat Benzoyl- $D, L$-arginin-p-nitroanilid (BAPA) $p$-Nitroanilin frei, dessen Absorption bei $405 \mathrm{~nm}\left(\epsilon=970 \mathrm{~m}^{2} / \mathrm{mol}\right)$ kontinuicrlich gemessen wird (10). Aus der Absorptionsdifferenz gegenüber dem "Leerwert von Trypsin" (Reagenziengemisch ohne Probe) läßt sich die Aktivität von $\alpha_{1}$-Antitrypsin in Inhibitoreinheiten pro Liter Serum oder Plasma (IU/1) berechnen:

Einc Inhibitoreinheit $\alpha_{1}$-Antitrypsin ist die Aktivität, welche eine Trypsineinheit hemmt. Eine Trypsineinheit ist die Enzymaktivität, welche bei $25^{\circ} \mathrm{C} 1 \mu \mathrm{mol}$ Substrat in $1 \mathrm{~min}$ umsetzt.

\section{Reagenzien, Methọden, Geräte}

$\alpha_{1}$-Antitrypsin, Farbtest (Best.Nr. 416509) sowie Testpackungen zur Bestimmung von $L$ - $\gamma$-Glutamyl-transferase (EC 2.3.2.2), Harnstoff, Triglyceriden und Gesamtprotein entstammten dem Lieferprogramm von Boehringer Mannheim GmbH, D-6800 Mannheim 31. Zur Bestimmung von Aspartat-aminotransferase (EC 2.6.1.1) und Alanin-aminotransferase (EC 2.6.1.2) wurden Packungen von E. Merck, D-6100 Darmstadt 2, eingesetzt. Kreatinin wurde mit der Jaffé-Reaktion kinetisch mit dem CreatininAnalyzer von Beckman Instruments, D-8000 München 40, gemessen. Nach der Arbeitsanleitung wurde $\alpha_{1}$-Antitrypsin kontinuierlich bei $25^{\circ} \mathrm{C}$ an einem Photometer Eppendorf $1101 \mathrm{M}$ (Netheler und Hinz, D-2000 Hamburg) mit Thermostat, Wechselau tomatik und Schreiber bestimmt. Die Proteinelektrophorese wurde als Mikrozonenelektrophorese auf Celluloseacetatfolie, Färbung mit Ponceau S und automatischer Auswertung mit Elscript III (Hirschmann D-8000 München), durchgeführt.

Mit Natriumcitratlösung beschickte Probenröhrchen $(0,5 \mathrm{ml}$, $0,11 \mathrm{~mol} / 1$, Gesamtvolumen $5 \mathrm{ml}$ ) wurden von der $\mathrm{Fa}$. Sarstedt, D-5223 Rommelsdorf, bezogen (Best.Nr. 05268).

\section{Probanden}

Das Ausgangskollektiv der Erwachsenen $(N=100)$ bestand aus Blutspendern der Blutbank der Universitätsklinik Freiburg/Brsg. Per Anamnese wurden erfaßt: Alter, Geschlecht, Einnahme von Kontrazeptiva, prä- bzw. postprandialer Zustand, Rauchen. Schwangere sowie Probanden mit aktuellen Erkrankungen oder Medikamenten-Therapie sind im Ausgangskollektiv nicht enthalten. Zur Auswahl eines Referenzkollektivs wurden klinischchemische Kenngrößen eingesetzt, welche bei Leber-, Nierensowie entzündlichen Erkrankungen verändert sind.

Referenzwerte: Aspartat-aminotransferase: Männer bis $18 \mathrm{U} / \mathrm{l}$. Frauen bis $15 \mathrm{U} / \mathrm{l}$; Alanin-aminotransferase: Männer bis $22 \mathrm{U} / \mathrm{l}$, Frauen bis $17 \mathrm{U} / \mathrm{l} ; L-\gamma$-Glutamyltransfcrase: Männer 6-28 U/1. Frauen 4-18 U/1; Harnstoff: $1,7-8,3 \mathrm{mmol} / \mathrm{l}$; Kreatinin: Männer 44-97 $\mu \mathrm{mol} / 1$, Frauen 44-80 $\mu \mathrm{mol} / \mathrm{l}$; Albumin 38-54 g/l, $\alpha_{1}$-Globulin $1-3 \mathrm{~g} / 1, \alpha_{2}$-Globulin $4-9 \mathrm{~g} / 1, \beta$-Globulin $5-11 \mathrm{~g} / \mathrm{l}$, $\gamma$-Globulin 6-16 g/l.

\section{Probennahme und Qualitätssicherung}

Die Blutabnahme erfolgte vormittags. Nach Lösen der Stauung wurden etwa $500 \mathrm{ml}$ Blut (Blutkonserve) und danach Blut für Serum- und Plasmagewinnung entnommen. Citratplasma und Serum wurden durch $20 \mathrm{~min}$ Zen trifugation bei $2000 \mathrm{~g}$ erhalten. Die Proben wurden bei $-20^{\circ} \mathrm{C}$ eingefroren und innerhalb von 4 Wochen untersucht.
Die Präzision der Meßergebnisse von $\alpha_{\AA}$-Antitrypsin wurde mit den Humancitratplasmen PreciChrom ${ }^{\circledR}$ I und II (Charge 09001) sowie cinem portioniert eingefrorenen laboreigenen Plasma- und Serum-Pool überprüft. Die Wiederfindung wurde mit PreciChrom ${ }^{\circledR}$ I und II (Charge 1002) überprüft, indem an 10 Tagen jeweils Doppelbestimmungen in beiden Kontrollplasmen durchgeführt wurden.

$\therefore 1$

\section{Statistische Methoden und Berechnungen}

Die Präzision in der Serie und von Serie zu Serie wurde mit Hilfe einer einfachen Varianzanalyse berechnet. Die Verteilung der Meßwerte wurde durch Angabe der Perzentile beschrieben. Zur Überprüfung eines geschlechtsspezifischen Unterschiedes sowie anderer Einflußgrößen auf die $\alpha_{1}$-Antitrypsin-Aktivität (siehe Tab. 4) wurde der U-Test von Mann-Whitñey eingese tzt (11). Als Irrtumswahrscheinlichkeit wurde $\alpha=0,05$ festgelegt.

\section{Berechnungen für Réferenzwerte}

$\alpha_{1}$-Antitrypsin in Serum und Citratplasma aus $9+1$ verd. Blut:

$\mathrm{kIU} / \mathrm{l}\left(25^{\circ} \mathrm{C}\right)=26,03 \cdot \Delta \mathrm{A} / \mathrm{min}$. $\mathrm{g} / \mathrm{l}\left(25^{\circ} \mathrm{C}\right)=36,50 \cdot \Delta \mathrm{A} / \mathrm{min}$.

Nach Angabe des Herstellers wurde der Faktor für die Angabe in $\mathrm{g} / \mathrm{l}$ durch Vergleich der photome trischen Aktivitätsbestimmung mit der radialen Immunodiffusion erhalten.

\section{Berechnungen für Vergleichsuntërsuchungen Plasma/Serum}

Die jeweilige Verdünnung des Blutes bei Verwendung von Citratlösung als Antịọgulans hängt vom individuellen Hämatokrit (PCV) ab.

Damit gilt:

$\alpha_{1}$-Antitrypsin in Plasma:

$$
\mathrm{kIU} / 1\left(25^{\circ} \mathrm{C}\right)=26,03 \cdot\left[1+\frac{1}{9(1-\mathrm{PCV})}\right] \cdot \Delta \mathrm{A} / \mathrm{min}
$$

\section{Ergebnisse und Diskussion}

\section{Qualitätssicheruing}

Tabelle 1 enthält die Ergebnisse der Qualitätssicherung von $\alpha_{1}$-Antitrypsin in Kontrollplasmen sowie eingefrorenen und einmal wiederaufgetauten Plasma- und Serumproben. Die Präzision in der Serie und von Serie zu Serie war gut bis befriedigend (VK: 1,4 bis 6,5\%); die Wiederfindung des Sollwertes war während des Untersuchungszeitraumes gegeben.

\section{Referenzwerte}

In Tabelle 2 ist die Verteilung der $\alpha_{1}$-AntitrypsinAktivitäten sowie -Konzentrationen im Ausgangskollektiv $(N=100)$ für Serum und Plasma wiedergegeben. Für Serum bzw. Citratplasma (aus $9+1$ verd. Blut) ergeben sich nur geringe Unterschiede: Wählt man zur Charakterisierung der Variabilität der Meßwerte den $90 \%$ :Bereich zwischen dem 5. und 95. Pérżentil, dann unterscheidet sich - bedingt durch die unterschiedliche Rechtsschiefe der Verteilungen - lediglich die Obergrenze. Die klinisch-wichtigere Untergrenze des Referenzbereiches liegt bei $1,4 \mathrm{kIU} / \mathrm{l}$ entsprechend $1,9 \mathrm{~g} / 1$. 
Die Abbildung 1 zeigt die Verteilungen für Serum und Plasma. Wie die Verteilungen der in Tabelle 3 dargestellten klinisch-chemischen Kenngrößen zeigen, enthält das Ausgangskollektiv Probanden, bei denen Erkrankungen nicht auszuschließen sind. Setzt man die jeweiligen Referenzwerte als Auswahlkriterien an, dann ergibt sich eine Reduzierung des Kollektivs um 15 auf 85. Zwischen dem Endkollektiv $(N=85)$ und dem aussortierten Kollektiv $(\mathrm{N}=15)$ besteht jedoch bezüglich der $\alpha_{1}$-Antitrypsin-Werte kein Unterschied (Tab. 4), so daß das Ausgangskollektiv zugleich als Referenzkollektiv angesehen werden kann.

Alter, Geschlecht, Rauchen oder prandialer Zustand beeinflussen offensichtlich die $\alpha_{1}$-Antitrypsin-Aktivität nicht (Abb. 2, Tab. 4). Dagegen zeigen Frauen, die hormonale Kontrazeptiva einnehmen, signifikant höhere Werte als Frauen ohne hormonale Kontrazeption. Da der Stichprobenumfang der beiden Kollektive sehr klein ist, kann aus diesem Befund keine Schlußfolgerung über eine Differenzierung der Referenzwerte gezogen werden. Von den in der Literatur beschriebenen Abhängigkeiten $(1,6-9)$ können wir damit Angaben über erhöhte $\alpha_{1}$-Antitrypsin-Werte bei hormonaler Kontrazeption bestätigen. Eine Geschlechts- oder Altersabhängigkeit geht aus unseren Daten nicht hervor.

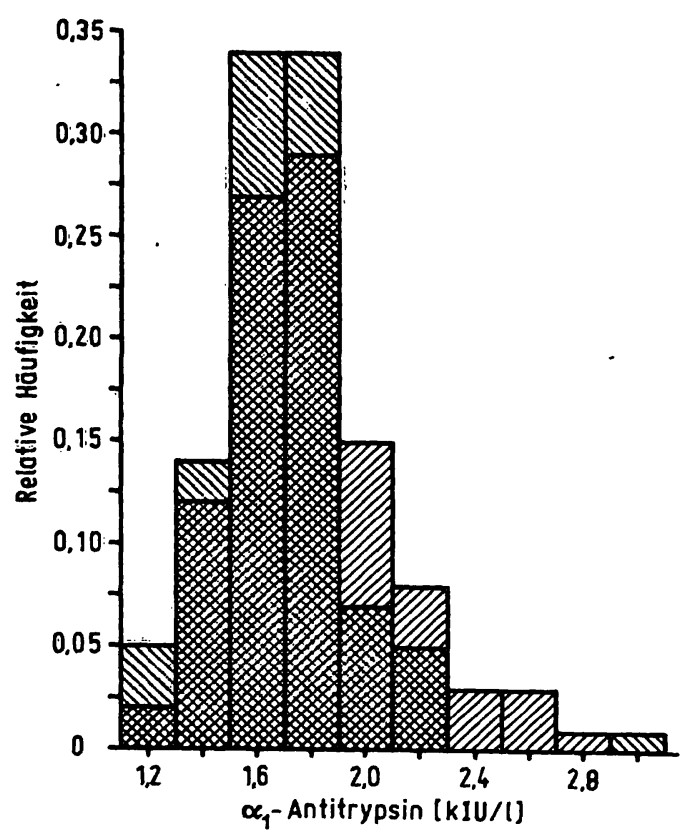

Abb. 1. Verteilung der $\alpha_{1}$-Antitrypsin-Aktivitäten $\left(25^{\circ} \mathrm{C}\right)$ in Serum and Plasma im Ausgangskollektiv ( $N=100 \mathrm{Er}$ wachsene) $\alpha_{1}$-Antitrypsin (Serum) [kIU/l] $N_{1}=100 \mathbb{Z}$ $\alpha_{1}$-Antitrypsin (Plasma) [kIU/1] N $\mathbf{N}_{2}=100 \mathbb{N}$ Die Plasmawerte beziehen sich auf $9+1$ verd. Blut

Tab. 1. $\alpha_{1}$-Antitrypsin: Präzision in Kontrollplasmenen, Nativplasma und Serum sowie Wiederfindung in Kontrollplasmen.

\begin{tabular}{|c|c|c|c|c|c|c|c|c|c|c|c|c|}
\hline \multirow{2}{*}{$\begin{array}{l}\text { Unter- } \\
\text { suchungs- } \\
\text { material }\end{array}$} & \multicolumn{3}{|c|}{ Peṛzentile $\left.^{1}\right)[\mathrm{kIU} / \mathbf{1}]$} & \multicolumn{2}{|c|}{ VK [\%] } & \multirow{2}{*}{$\begin{array}{l}\text { Kontroll- } \\
\text { plasmen }\end{array}$} & \multirow{2}{*}{$\begin{array}{l}\text { Sollwertt } \\
\text { [kIU/1] } \\
\text { (Streu- } \\
\text { bereich }^{2} \text { ) }\end{array}$} & \multicolumn{5}{|c|}{ Perzentile $^{3}$ ) [kIU/l] } \\
\hline & Min. & Median & Max. & Serie & $\begin{array}{l}\text { Serièel } \\
\text { Serie }\end{array}$ & & & Min. & $10 \%$ & $50 \%$ & $90 \%$ & Max. \\
\hline PreciChrom ${ }^{\circledR}$ I & 1,19 & 1,41 & $1,5 \dot{5}$ & 3,4 & 6,5 & PreciChrom $^{\circledR}$ I & $\begin{array}{l}1,17 \\
(0,94-1,40)\end{array}$ & 1,10 & 1,15 & 1,19 & 1,33 & 1,36 \\
\hline PreciChrom ${ }^{\circledR}$ II & 0,70 & $0,7 j$ & 0,84 & 5,2 & 2,7 & PreciChrom $^{\circledR}$ II & $\begin{array}{l}0,61 \\
(0,49-0,73)\end{array}$ & 0,54 & 0,54 & 0,61 & 0,70 & 0,70 \\
\hline Nativiplasma & 1,38 & 1,49 & 1,64 & 2,2 & $4 ; 1$ & & & & & & & \\
\hline Serum & 2,16 & 2,40 & 2,58 & 1,4 & 5,4 & & & & & & & \\
\hline
\end{tabular}

1) Niedrigste und höchste Weṛte sowie Mediane an 5 Tagen mịit jeweils 6 Bestimmungen $(N=30)$.

2) Der Streubereich entspricht $\pm 20 \%$ des vom Hersteller angegebenen Sollwertes.

3) Die Werte geben das 10., 50. (Median), 90. Perzentil sowie ñiedíigste und höchste Mệuerte für Doppelbestimmungen an 10 Tagen $(\mathrm{N} \equiv 20)$ añ.

Tab. 2. Verteilung der $\alpha_{1}$-Antitrypsin-Werte $\left(25^{\circ} \mathrm{C}\right)$ im Ausgañgskollektiv.

Die Werte geben das 2,5., 5., 50. (Median), 95. und 97,5. Perzentil sowie niedrigste und höchste Meßwerte in verschiedenen Probenmaterialien für 100 Probạnden an.

\begin{tabular}{rllllllll}
\hline$\alpha_{1}$-Antitrypsin & Probenmaterial & Minimum & $2,5 \%$ & $5 \%$ & $50 \%$ & $95 \%$ & $97,5 \%$ & Maximum \\
\hline kIU/1 & Serum & 1,2 & 1,3 & 1,4 & 1,8 & 2,4 & 2,6 & 2,9 \\
g/1 & Serum & 1,6 & 1,9 & 1,9 & 2,5 & 3,4 & 3,6 & 4,1 \\
kIU/1 & Pläsma verd. $^{1}$ ) & 1,2 & 1,3 & 1,4 & 1,7 & 2,1 & 2,2 & 3,0 \\
g/1 & Plasma verd. $\left.^{1}\right)$ & 1,7 & 1,8 & 1,9 & 2,3 & 3,0 & 3,1 & 4,2 \\
kIU/1 & Plasma $^{2}$ ) & 1,4 & 1,5 & 1,6 & 2,0 & 2,6 & 2,6 & 3,6 \\
\hline
\end{tabular}

1) Die Werte beżiehen sich auf Citratplasma aus $9+1$ verd. Blut.

${ }^{2}$ ) Dex experimentell ermittelte Wert für Citratplasma aus $9+1$ verd. Blut wurde über den individuellen Hämatokrit korrigiert, d.h. auf unverdünntes Plasma bezogen. 


\section{Unterschiede Plasma/Serum}

Berücksichtigt man bei der Berechnung der Aktivitäten im Plasma die jeweilige Verdünnung mit Citratlösung, d.h. den individuellen Hämatokrit, dann ergeben sich für Plasma höhere Aktivitäten als für Serum (Tab. 2). Die Differenzen zwischen Plasma und Serum liegen zwischen $-0,02$ und 0,32 Anteilen (5. bzw. 95. Perzentil), der Median liegt bei 0,13 Anteilen (13\%; Abb. 3).

Diese Unterschiede lassen sich möglicherweise damit erklären, daß es bei der Gerinnung zu einer Freisetzung von Proteinasen kommt und zwischen $\alpha_{1}$-Antitrypsin und den aktiven Proteinasen Komplexe entstehen. Denkbar wäre auch eine Fixierung an das Fibrin-Gerinnsel. Ein Teil des $\alpha_{1}$-Antitrypsin steht damit zur Komplexbildung mit Trypsin nicht mehr zu Verfugung, und man findet im Serum niedrigere Werte als im Plasma.

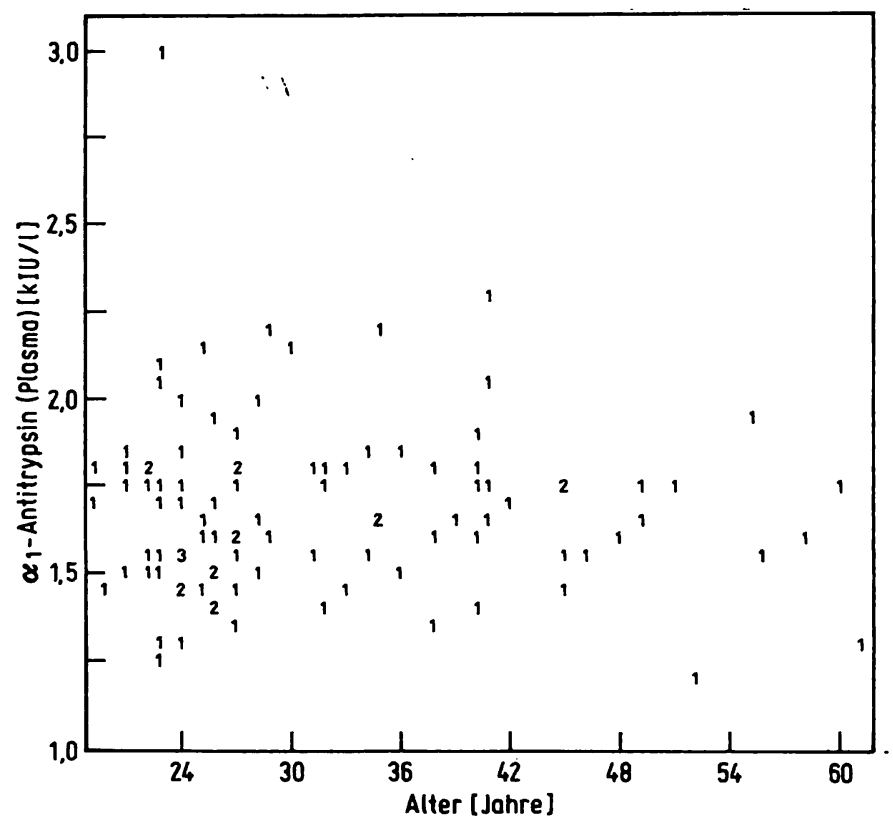

Abb. 2. Häufigkeitsverteilung der $\alpha_{1}$-Antitrypsin-Aktivitäten (Plasma) im Ausgangskollektiv ( $=100)$ in Abhängigkeit vom Alter.

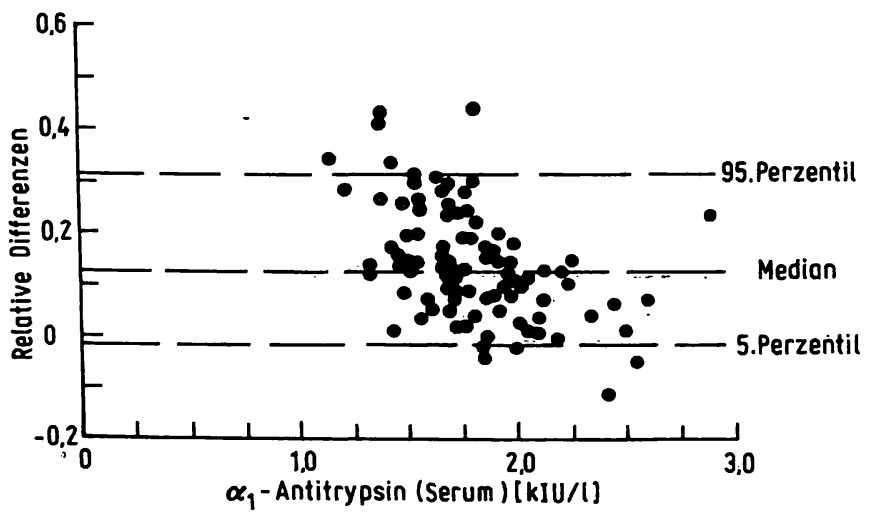

Abb. 3. $\alpha_{1}$-Antitrypsin: Relative Differenzen zwischen den Aktivitätsmessungen in Plasma und Serum $(\mathrm{N}=100)$, bezogen auf Serum.

\section{Danksagung}

Für die sorgfältige Durchführung der Untersuchungen danken wir Frau H. Flad und Frau B. Wohlhüter.

Tab. 3. Verteilung klinisch-chemischer Kennggößen im Aụsgạngskollektiv.

Die Werte geben das 5., 50. (Median) und 95. Perzentil sowie niedrigste und höchste Meßwerte von 100 Probanden an.

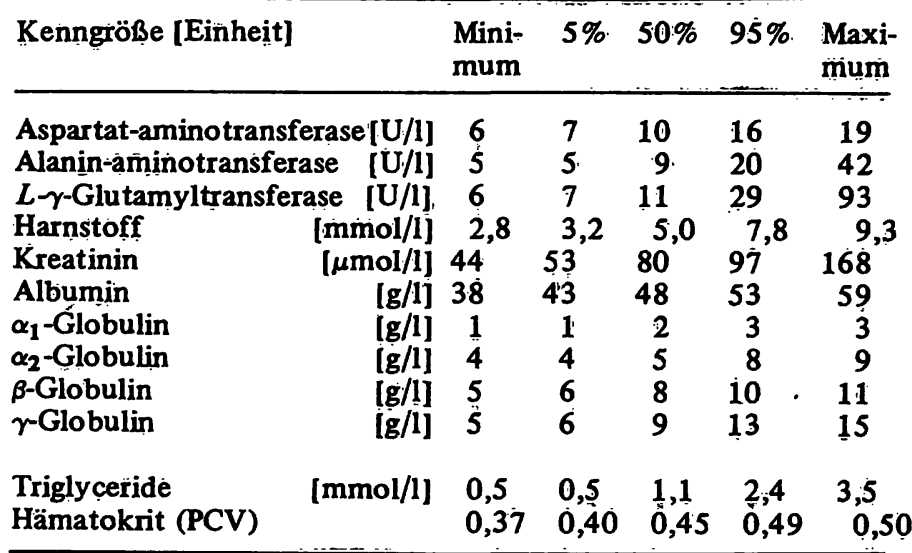

Tab. 4. Verteilung der $\alpha_{1}$-Antitrypsin-Aktivitäten $\left(25^{\circ} \mathrm{C}\right)$ in verschiedenen Kollektiven.

Dargestellt sind von $\mathbf{N}$ Probanden die niedrigsten, höchșsten und mittleren Aktivitäten (Mediane) in Citratplasma aus $9+1$ verd. Blut sowie die Ergebnisse der Signifikanzprüfung mit dem U-Test.

\begin{tabular}{|c|c|c|c|c|c|}
\hline \multirow[t]{2}{*}{ Gruppe } & \multirow[t]{2}{*}{$\mathbf{N}$} & \multicolumn{3}{|c|}{$\alpha_{1}$-Antitrypsin [kIU/l] } & \multirow{2}{*}{$\begin{array}{l}\text { Signị- } \\
\text { fikanz } \\
(\alpha \doteq 0,05)\end{array}$} \\
\hline & & $\begin{array}{l}\text { Mini- } \\
\text { mum }\end{array}$ & Median & $\begin{array}{l}\text { Maxi- } \\
\text { mum }\end{array}$ & \\
\hline $\begin{array}{l}\text { Endkollektiv } \\
\text { aussortiertes Kollektiv }\end{array}$ & $\begin{array}{l}85 \\
1.5\end{array}$ & $\begin{array}{l}1,2 \\
1,4\end{array}$ & $\begin{array}{l}1,7 \\
1,6\end{array}$ & $\begin{array}{l}3,0 \\
2,3\end{array}$ & $\underline{\mathbf{b}}$ \\
\hline $\begin{array}{l}\text { Männer } \\
\text { Frauen }\end{array}$ & $\begin{array}{l}76 \\
24\end{array}$ & $\begin{array}{l}1,2 \\
1,3\end{array}$ & $\begin{array}{l}1,7 \\
1,7\end{array}$ & $\begin{array}{l}2,3 \\
3,0\end{array}$ & keine \\
\hline $\begin{array}{l}\text { Raucher } \\
\text { Nichtraucher }\end{array}$ & $\begin{array}{l}61 \\
39\end{array}$ & $\begin{array}{l}1,3 \\
1,2\end{array}$ & $\begin{array}{l}1,7 \\
1,6\end{array}$ & $\begin{array}{l}3,0 \\
2,2\end{array}$ & keine \\
\hline $\begin{array}{l}\text { Keine hormon. Kontra- } \\
\text { zeptiva }\end{array}$ & 17 & 1,3 & 1,5 & 1,9 & $\begin{array}{l}\text { Signi- } \\
\text { fikanz }\end{array}$ \\
\hline $\begin{array}{l}\text { Hormonale Kontra- } \\
\text { zeptiva }\end{array}$ & 7 & $1,8$. & 2,1 & 3,0 & \\
\hline $\begin{array}{l}\text { Nüchtern } \\
\text { Post-prandial }\end{array}$ & $\begin{array}{l}32 \\
68\end{array}$ & $\begin{array}{l}1,3 \\
1,2\end{array}$ & 1,7 & $\begin{array}{l}2,3 \\
3,0\end{array}$ & keine \\
\hline $\begin{array}{l}\text { Triglyceride norimal } \\
\text { Triglyceride erhöht }{ }^{\mathrm{a}}\end{array}$ & $\begin{array}{l}88 \\
12\end{array}$ & $\begin{array}{l}1,2 \\
1,3\end{array}$ & $\begin{array}{l}1,7 \\
1,6\end{array}$ & $\begin{array}{l}3,0 \\
2,2\end{array}$ & b \\
\hline $\begin{array}{l}\text { Alter: }<35 \text { Jahre } \\
\text { Alter: } \geqslant 35 \text { Jahre }\end{array}$ & $\begin{array}{l}66 \\
34\end{array}$ & $\begin{array}{l}1,3 \\
1,2\end{array}$ & $\begin{array}{l}1, \overline{7} \\
1,7\end{array}$ & $\begin{array}{l}3,0 \\
2,3\end{array}$ & keine \\
\hline
\end{tabular}

a mehr als 2,0 minol/1.

b Wegen der unterschiedlichen Fallzahlen der Kollektive wurde keine Signifikanzprüfung durchgeführt. Dà der Bereich des Zweitkollektivs innerhalb des Wertebereiches des Erstkollektivs liegt, dürfte jedoch kein Untersschied zwischen beiden Gruppen vorliegen. 


\section{Literatur}

1. Editorial (1973) Brit. Med. Journ. 1, 758.

2. Laurell, C. B. \& Eriksson, S. (1963) Scand. J. Clin. Lab. Invest. 15, 132-140.

3. Sharp, H. L., Bridges, R. A., Krivit, W. \& Freier, E. F. (1969) J. Lab. Clin. Med. 73, 934-939.

4. Karitzky, D., Lesch, R., Goedde, H. W., Witt, 1.,Boehm, N., Beckmann, R., Jobke, A. \& Künzer, W. (1978) Dtsch. Med. Wochenschr. 103, 161-166.

5. Karitzky, D. \& Witt, I. (1979) Therapiewoche 29, 8622 -8624 .

6. Herbeth, B., Bagrel, A., Dalo, B., Siest, G., Leclerc, J. \& Rauber, G. (1980) Clin. Chim. Acta 112, 293-299.
- 7. Liappis, N., Beeser, H., Fritsche, Ch. \& Hildenbrand, G.

$$
\text { (1981) Lab. Med. 5, 73-78. }
$$

8. Denko, Ch. W. \& Gabriel, P. (1981) Ann. Clin. Lab. Sci. 11, 63-68.

9. Liappis, N., Schlebusch, H. \& Hildenbrand, G. (1981) Klin. Pädiat. 193, 88-90.

10. Hoffmann, M. L., van den Broek, W. G. M. \& Jansen, A. P. (1976) Clin. Chim. Acta 71, 251-259.

11. Conover, W. J. (1971) Practical nonparametric statistics J. Wiley \& Sons, New York, pp. 224-236.

Dr. rer. nat. W. Tritschler

Boehringer Mannheim GmbH

Diagnostica Forschung

Sandhofer Straße 116

D-6800 Mannheim 31

Prof. Dr. Irene Witt

Klinisch-Chemisches und Biochemisches Labor Universitäts-Kinderklinik

Mathildenstraße 1

D-7800 Freiburg 